Articulación entre los sistemas de pregrado y grado de la UNLP. La experiencia del bachillerato en Saneamiento y Gestión Ambiental

Ana María García Munitis, Lucila Benito

Revista ES (en y sobre Educación Superior)

Vol.1, N¹-2 / Fecha de publicación: 27/12/2021

e-ISSN: $2718-6539$

\title{
https://revistas.unlp.edu.ar/ES/index
}

IIES - Facultad de Odontología

DOI: https://doi.org/10.24215/27186539e016

\section{Articulación entre los sistemas de pregrado y grado de la UNLP}

La experiencia del bachillerato en Saneamiento y Gestión Ambiental

Articulation between the undergraduate and graduate systems of the UNLP

The experience of the baccalaureate in Sanitation and Environmental Management

Articulação entre os sistemas de graduação e pós-graduação da UNLP A experiência do bacharelado em Saneamento e Gestão Ambiental 
Directora del Colegio Nacional "Rafael Hernández" - UNLP anagarciam@ciudad.com.ar

\author{
Lucila Benito
}

lucilabenito@yahoo.com.ar

\title{
Resumen
}

Este trabajo da cuenta del inicio de la implementación de una Secundaria Técnica impulsada por el Colegio Nacional Rafael Hernández y la Facultad de Ingeniería de la Universidad Nacional de La Plata (UNLP). La iniciativa repiensa el sistema pregrado y crea otras formas de pasaje entre niveles educativos que contemplen las especificidades del contexto, las problemáticas emergentes y las complejidades del tránsito abrupto de una instancia a la otra. En este sentido, se promueve la articulación institucional entre ambas unidades académicas con el fin de abordar de manera integral dicho desafío. La escuela propone entonces una modalidad orientada al Saneamiento y Gestión Ambiental. Este proyecto surgió para dar respuesta a múltiples problemáticas sociales, económicas y culturales que muchos/as estudiantes enfrentan en el pasaje entre niveles educativos y en el tránsito por cada uno de ellos. Además, implica una política de intervención y gestión en la inclusión de una multiplicidad de actores/as en el entramado relacional de la UNLP. 


\section{Abstract}

This work gives an account of the beginning of the implementation of a Technical Secondary promoted by the Rafael Hernández National College and the Faculty of Engineering of the National University of La Plata (UNLP). The initiative rethinks the undergraduate system and creates other forms of passage between educational levels that address the specificities of context, emerging problems and the complexities of abrupt transit from one instance to the other. In this sense, institutional articulation between the two academic units is promoted in order to address this challenge in a comprehensive way. The school then proposes a modality oriented to Sanitation and Environmental Management. This project emergedto respond to multiple social, economic and cultural problems that many students face in the passage between educational levels and in transit through each of them. In addition, it implies a policy of intervention and management in the inclusion of a multiplicity of actors in the relational fabric of UNLP.

\section{Resumo}

Este trabalho dá conta do início da implementação de um Secundário Técnico promovido pelo Colégio Nacional Rafael Hernández e pela Faculdade de Engenharia da Universidade Nacional de La Plata (UNLP). A iniciativa repensa 
o sistema de licenciatura e cria outras formas de passagem entre os níveis educativos que abordam as especificidades do contexto, os problemas emergentes e as complexidades do trânsito abrupto de um caso para o outro. Neste sentido, promove-se a articulação institucional entre as duas unidades académicas, a fim de abordar este desafio de forma abrangente. A escola propõe então uma modalidade orientada para o Saneamento e Gestão Ambiental. Este projeto surgiu para responder a múltiplos problemas sociais, económicos e culturais que muitos estudantes enfrentam na passagem entre os níveis educativos e em trânsito através de cada um deles. Além disso, implica uma política de intervenção e gestão na inclusão de uma multiplicidade de intervenientes no tecido relacional da UNLP.

\section{Palabras clave}

Articulación, Escuela secundaria, Saneamiento, Ambiental

\section{Keywords}

Articulation, High School, Sanitation, Environmental

\section{Palavras-chave}

Articulação, Escola Secundária, Saneamento, Ambiente 


\section{Articulación institucional}

Este trabajo surge a partir del balance alrededor de la escuela secundaria, y en especial de un colegio de la UNLP con peso específico y destacado en la ciudad. En este sentido, a partir de lecturas del escenario educativo actual, podemos ver que se despliegan nuevas dinámicas sociales y culturales que nos hacen repensar el sentido y la función de la escuela secundaria hoy en día. Así, analizar la formación de los/as jóvenes como ciudadanos/as portadores de derechos, pensar el acceso al mundo del trabajo y la continuidad con estudios superiores deben ser comprendidos en vinculación con el contexto social actual para generar una gestión y política educativa acorde a los objetivos de gestión, haciendo que estos se piensen en relación dinámica con las necesidades de la época.

A partir de esto nos propusimos reflexionar sobre la experiencia específica de la articulación entre dos unidades académicas de la UNLP: el Colegio Nacional y la Facultad de Ingeniería, desde la gestión del primero. Nuestra mirada se coloca tanto en el pasaje de un nivel a otro como en los aprendizajes que los/as estudiantes necesitarían para verse fortalecidos en sus trayectorias y transitar con éxito su escolaridad y educación en cada nivel.

Asumimos entonces que la escuela secundaria es una institución que ocupa un lugar central para la formación y participación ciudadana, capaz de revalorizar entre los/as jóvenes de hoy el aprendizaje y el conocimiento, para comprender 
y responder a las problemáticas sociales e individuales que atraviesan. Implica también potenciar el desarrollo de sus cualidades y capacitarlos/as para que puedan continuar su desenvolvimiento intelectual y laboral.

La experiencia es novedosa ya que no existía previamente formación técnico profesional en el Colegio Nacional "Rafael Hernández". Esto trae consigo la necesidad de pensar nuevas estrategias de enseñanza, objetivos específicos y un accionar conjunto de la UNLP que resulta muy enriquecedor. Pero sobre todo, porque permite pensar estas vinculaciones en torno a una formación específica, técnica, que resulta de notorio aporte y relevancia para distintos territorios de la ciudad.

\section{El desafío de la Universidad}

Partimos así de la Ley Nacional de Educación No 26.206, que establece entre sus fines y objetivos de política educativa la obligatoriedad de la enseñanza secundaria, comprendida como educación integral y de calidad, que debe garantizar la inclusión, la igualdad de oportunidades en el acceso, permanencia y egreso de los/as estudiantes. Por otra parte, y en concordancia con la Ley, el Plan Estratégico de la UNLP 2018-2022 incluye entre sus objetivos para la enseñanza de pregrado la calidad de la enseñanza, permanencia, articulación entre niveles, extensión y transferencia e innovación de los modelos institucionales. 
El contrato fundacional de los colegios dependientes de la UNLP incluye la experimentación, innovación y transferencia como base de los mismos. Cada establecimiento fue concebido como una planta piloto de investigación y experimentación, como "escuelas modelo" o innovadoras. Esta visión, que no siempre ha sido sostenida y desarrollada a lo largo de la historia institucional, debe ser asumida y resignificada en el contexto actual. Pensar una escuela que fomente la investigación educativa, el intercambio de ideas, prácticas pedagógicas y la innovación, es pensar en un proyecto educativo que instala la necesidad y posibilidad del cambio como el motor generador de la transformación educativa y social. Entonces, no debe encerrarse en el cerco institucional sino constituirse en un saber susceptible de ser transferido a otras instituciones educativas, es decir extensible a otras realidades y dinámicas institucionales y pedagógicas con su respectiva adecuación.

Para enfrentar estos desafíos, atendiendo al carácter experimental que la UNLP le ha asignado al Sistema de Pregrado, surgió la propuesta de pensar la creación de un bachillerato técnico, en un acuerdo de articulación entre el Colegio Nacional Rafael Hernández y la Facultad de Ingeniería de la UNLP. Esta escuela fue pensada entonces como una nueva modalidad del Colegio Nacional, orientada en Saneamiento y Gestión Ambiental.

En este sentido, se propuso pensar un/a nuevo/a bachiller que no plantease únicamente un nuevo plan de estudios sino que prescribiera los elementos que 
lo relacionan y articulan con el mundo del trabajo y la producción, respetando la pertinencia al medio y promoviendo el desarrollo de los territorios que la contienen, así como también a sus habitantes. De allí se desprende la posibilidad de brindar jóvenes especializados/as en aquellos temas y problemáticas que tienen relación con cursos de agua, cuencas, zonas inundables, agua potable, cloacas y manejos de residuos.

Señalamos la relevancia de impulsar esta iniciativa desde la Universidad (pública y gratuita) como el aprovechamiento de su estructura institucional para el abordaje de estos temas, entendiendo que las escuelas provinciales no cuentan, en este contexto, con esa especialidad. La propuesta implica entonces un proyecto de inclusión social, cultural y educativa, pero además, el fortalecimiento de las distintas ramas de formación y actividad de la UNLP, entendiendo que no solo se incorporan a estudiantes desde otra mirada de la escuela secundaria, sino también que se potencian nuevos conocimientos y aplicaciones territoriales en aportes a la ciudad y el ambiente. Esto es entonces un desafío que potencia a las instituciones específicas que lo implementan, así como a sus alrededores y a la ciudadanía en general.

El compromiso asumido frente a este nuevo ordenamiento impulsa a formar jóvenes que puedan desempeñarse como actores/as en procesos productivos en pequeñas y medianas empresas o industria nacional. También como emprendedores autogestivos con sentido crítico y de responsabilidad 
ciudadana que sean verdaderos agentes de promoción del cambio y el desarrollo, local, provincial y nacional. Se conforma de esa forma una alternativa de educación obligatoria, con seis años de duración, y se constituye como una unidad pedagógica y organizativa comprendida por una formación común y una orientada, de carácter diversificado, que responde a diferentes áreas del conocimiento, del mundo social y del trabajo.

\section{Gestión ambiental y saneamiento}

Formar jóvenes con una especificidad en saneamiento y gestión ambiental, constituye un recurso humano indispensable para el desarrollo local, además de poder realizar aportes a la generación del conocimiento y la innovación tecnológica.

La consideración de la temática ambiental en el ámbito urbano y en el rural, desde una perspectiva global, es un fenómeno nuevo al menos en América Latina. Desde hace varias décadas los esfuerzos por mejorar las condiciones ambientales en nuestras ciudades se limitaron a la construcción progresiva del sistema de captación de agua y transporte de aguas servidas, sistemas de recolección de basura, entre otros.

La salud de una comunidad está directamente relacionada con factores que condicionan la relación entre salud y enfermedad, y la necesidad básica humana de un ambiente seguro. La salud ambiental ofrece el marco conceptual 
para lograr mejores condiciones desde el punto de vista sanitario, ambiental y socioeconómico, y para esto incorpora múltiples sectores y disciplinas. El saneamiento básico y la calidad ambiental son dos pilares fundamentales para el mejoramiento de las condiciones de vida de una población.

En el área de saneamiento básico se contemplan aquellas actividades relacionadas con el mejoramiento de las condiciones básicas que afectan a la salud, es decir el abastecimiento de agua, la disposición de excretas, residuos sólidos, vivienda y control de la fauna nociva. Entre los componentes operativos del saneamiento básico se encuentran: el agua potable, el alcantarillado, la disposición de excretas en el medio rural, el aseo urbano, el mejoramiento de la vivienda, la protección de los alimentos, el control de la fauna nociva y el control de zoonosis.

En el área de calidad ambiental se indica la caracterización del impacto del desarrollo, como la contaminación ambiental y su efecto sobre la salud pública. Los componentes operativos de calidad ambiental refieren a siete rubros que involucran desafíos globales para la salud y el medio ambiente: alimentación, agricultura, agua, industria, asentamientos humanos y urbanización, y problemas transfronterizos e internacionales.

En este sentido, es importante vincular la educación y la enseñanza con las problemáticas ambientales del contexto que nos corresponde, así como soluciones y estrategias para un abordaje responsable. Hoy en día la 
educación ambiental es un proceso que pretende formar y crear conciencia a todos los seres humanos con su entorno, siendo responsables de su uso y mantenimiento (Rengifo, 2012).

Proponer el cruce entre educación y enseñanza con el conocimiento sobre ambiente y saneamiento es pensar políticamente la problemática y asumir la responsabilidad social y ciudadana. La educación ambiental es un proceso que busca reconocer valores y clarificar conceptos para fomentar prácticas, destrezas, habilidades y aptitudes necesarias que faciliten la vinculación comunitaria con el medio ambiente. Desde una propuesta de secundario obligatorio de seis años de duración sobre la temática con vinculación con las problemáticas locales, se pretende aportar a la comunidad con experiencia y conocimiento sobre el tema.

\section{Intervención}

El proyecto comparte los lineamientos generales del Plan Estratégico de la UNLP 2018-2022 en el sentido que establece la ley de la educación secundario o de pregrado e incluye como objetivos para estas instituciones el mejoramiento de la calidad de la enseñanza apuntando a prácticas de retención e inclusión escolar y prácticas de innovación de enseñanza como así también a la promoción de la investigación y extensión. Tiene como propósito responder a la demanda de la sociedad, promoviendo la equidad social, 
custodiando la igualdad de oportunidades y mejorando la calidad de la oferta pública en la enseñanza de pregrado.

Ya en nuestro Proyecto del año 2014 sosteníamos que a treinta años de la puesta en vigencia del ingreso sin examen, no cabe duda que ha sido la mejor garantía de la igualdad de oportunidades educativas. El desafío era y continúa siendo, generar condiciones reales de permanencia y terminalidad. Más allá de las variadas propuestas que se han ido ensayando, los problemas del ingreso a la Escuela Secundaria siguen siendo los altos índices de repitencia y deserción en los primeros años. En este sentido, deberíamos plantearnos no sólo el tránsito de un nivel a otro sino también la posibilidad de que los alumnos transiten con éxito el nivel. Por eso, más allá de las valoraciones que puedan realizarse, y considerando que las propuestas hechas hasta el momento son acotadas, poco acordes al contexto en el que estamos inmersos, debemos repensar las formas de entender y atender la heterogeneidad.

La creación de esta experiencia de modalidad educativa es pensada como una articulación entre la Facultad de Ingeniería y el Colegio Nacional. Asume que la escuela secundaria es una institución que ocupa un lugar central para la formación y participación ciudadana, capaz de revalorizar entre los jóvenes de hoy el aprendizaje y el conocimiento, para comprender y responder a las problemáticas sociales e individuales por las que atraviesan; permitirles desarrollar sus cualidades y capacitarlos para que puedan continuar su 
desarrollo intelectual y humano. El Plan Estratégico de la UNLP 2018-2022 incluye entre sus objetivos para la enseñanza de pregrado, la calidad de la enseñanza, permanencia, articulación entre niveles, extensión y transferencia, e innovación de los modelos institucionales. Podemos destacar uno de los objetivos planteados por la gestión como guía de esta experiencia:

"Favorecer la inclusión, permanencia y egreso en todas las instancias, niveles, modalidades y ámbitos de formación, procurando superar los límites sociales de la exclusión y segmentación segregatoria, al mismo tiempo que se profundicen las políticas de bienestar y desarrollo educativo para todos los estudiantes". (Plan Estratégico de la UNLP 2018-2022, página 13)

En este sentido se piensa un nuevo bachiller imaginando no solamente un nuevo plan de estudios sino, prescribiendo los elementos que la relacionan y la articulan con el mundo del Trabajo y la Producción, respetando la pertinencia al medio y promoviendo el desarrollo de los territorios que la contienen y sus habitantes. Brindará también jóvenes especializados en todo lo que tiene que ver con cursos de agua, cuencas, zonas inundables, agua potable, cloacas, manejos de residuos, y las escuelas provinciales no cuentan con esa especialidad.

\section{Análisis situacional}


En el escenario educativo actual, se despliegan nuevas dinámicas sociales y culturales que implican re-pensar el sentido y la función de la escuela secundaria, en cuanto a la formación de los jóvenes como ciudadanos portadores de derechos, a favorecer el acceso al mundo del trabajo y a la continuidad de estudios superiores.

El potencial creativo de las escuelas técnicas es prácticamente infinito: vehículos eléctricos, saneamiento de acuíferos, biodigestores, celdas solares, materiales de construcción económicos, sillas de ruedas, vehículos inteligentes, soluciones para el hogar, reciclaje de materiales, son sólo algunos de los desarrollos y actividades que vienen realizando las escuelas técnicas día a día, desarrollos que, en general, se basan en soluciones novedosas y económicas que, en la mayoría de los casos, están pensados para cubrir demandas y necesidades productivas de las PyMES o brindar respuestas a acuciantes necesidades de las comunidades más vulnerables.

Por otra parte, la Educación Técnica, también, tiene la capacidad de contribuir significativamente a la movilidad social, ya que, dada la índole de sus titulaciones que acreditan competencias profesionales, brinda a sus egresados muchas más posibilidades de inserción en el mercado laboral que la Educación Secundaria tradicional, sin que su objetivo primordial, sea por eso, que los mismos tengan como único horizonte insertarse en dicho mercado, sino que 
este constituya una alternativa o respaldo para la continuación de estudios superiores.

Por eso entendemos que la Universidad no puede permanecer ajena a la Educación Técnica y debe involucrarse de lleno en la misma, creando y sosteniendo su propia Escuela Secundaria Técnica.

\section{Desarrollo}

En esta primera etapa se trabajó en articulación con la Dirección General de Cultura y Educación de la provincia de Buenos Aires quien fue la encargada de seleccionar las instituciones, invitando a las familias de los jóvenes de zonas vulnerables a que tuvieran la oportunidad de estudiar en el Colegio Nacional a través de una propuesta concreta y especifica con posibilidad de insertarse en el mercado laboral.

De esta experiencia se seleccionaron 19 (diecinueve) escuelas primarias radicadas en diferentes zonas de la ciudad de La Plata y participan 60 jóvenes. Dichas escuelas son: EP2- EP7- EP9- EP27- EP29- EP 30- EP52- EP59 -EP 68- EP76- EP92 -EP 63- EP73-EP 78-EP83- EP 50- EP71 - EP40- EP79.

En esta etapa, cohorte 2019, se seleccionaron 60 vacantes lo que equivale a dos cursos. Se establecen dos vacantes por cada escuela seleccionada. Si los aspirantes superan la cantidad de vacantes se realizará un sorteo. 


\section{Estructura curricular}

La estructura del plan está conformada por una Educación Secundaria Básica (E.S.B) que incluye 10, 20 y 3o, y una Educación Secundaria Específica (E.S.E) que incluye 4o, 50 y 6o. De esta forma, el Plan de Estudios del Bachillerato en Saneamiento y Gestión ambiental está conformado por 6 (seis) años con 66 (sesenta y seis) materias divididas en formación general básica $(B A)$, formación científica-tecnológica (EX) y formación técnico específica ( $E S)$, más dos trayectos de práctica profesional(PP) en los dos últimos años.

La Educación Secundaria Básica comprende 26 materias, la Formación Científica- Tecnológica comprende 17 materias y la Formación Técnica Específica comprende 21 materias.

Las materias teórico-prácticas tienen un régimen de cursadas anual de 1ro. a 3ro. año y a partir de 4to. Año se incorpora la cuatrimestralidad. La optatividad se incorpora en 6to. año con un conjunto de materias estructurada en 4 campos:

- Social (ambiente y sociedad, cultura y ambiente, economía y política ambiental, el valor estético del ambiente)

- De la Salud (Impacto sobre la salud; salud y ambiente laboral; y nutrición y ambiente)

- Del Derecho (derecho laboral; de la seguridad en el trabajo; normas calidad; ética profesional) 
- Tecnológico (Sistemas de Recolección, transporte y Tratamiento de líquidos cloacales; Calidad de agua y técnicas de control; Proyecto y computo de obras de saneamiento)

El trayecto de Práctica Profesional se incorpora en 5to y 6to. La jornada diaria se estructura en el turno vespertino en donde los alumnos cursan en el Colegio y en la Facultad de Ingeniería de 16 hs. a 21 hs.

\section{La puesta en práctica}

La puesta en práctica de esta experiencia educativa tuvo un comienzo de lo más alentador. Ingresaron al Colegio un total de 61 estudiantes de distintos puntos del Gran La Plata. Las articulaciones hacia dentro de la institución no fueron pocas, se dispusieron los distintos departamentos y áreas para la optimización de la calidad educativa y mejorar la estancia de estos estudiantes en el Colegio.

En este sentido, se trabajó de manera conjunta y coordinada entre la gestión institucional (tanto del Colegio como de la Facultad de Ingeniería), el Departamento de Orientación Educativa (DOE) y aquellos/as docentes y preceptores/as que estarían a cargo del estudiantado ingresante en el vespertino. Esto permitió un trabajo integral con una iniciativa nueva, con desafíos particulares por las particularidades de la propuesta del secundario en general. 
Se propuso entonces llevar a cabo una serie de estrategias pedagógicas, institucionales y socio-educativas para garantizar la permanencia de estos/as estudiantes dentro del Colegio a lo largo de los años. Así, se dispusieron viandas destinadas los/as jóvenes que lo necesitaran, acompañamiento durante el inicio de clases con mayor rigurosidad, y luego un seguimiento constante (sin caer en miradas paternalistas pero sí con respaldo institucional), y se garantizó un transporte específicamente destinado a los/as estudiantes que lo necesitaran para la vuelta a sus hogares, sobre todo atendiendo a la lejanía de los mismos y el horario de finalización de la cursada. Con todas estas medidas, se buscó paliar aquellas dificultades y limitaciones de la asistencia a clases o la continuación en el Colegio para garantizar la continuidad de estos/as estudiantes. En el caso de los materiales para el aula, el Colegio junto a la Facultad de Ingeniería y la UNLP en general, dio a disposición cualquier herramienta que fuese necesaria para utilizar en el espacio áulico. Así también cuadernos, lapiceras y todo material que fuese requerido, garantizando así un acceso igualitario a los útiles escolares.

Sabemos que un acceso igualitario a la educación no tiene que ver únicamente con los útiles escolares, la cercanía del hogar y el acceso a viandas, pero está claro que mejorar las condiciones alimentarias, de acceso y de materiales para los estudiantes es un piso básico para la permanencia. Sin embargo, y como respuesta a esta primera enunciación, fue y será fundamental este trabajo 
coordinado e integral de los distintos espacios socio-educativos y pedagógicos con los que cuenta el Colegio Nacional "Rafael Hernández "específicamente y la UNLP en general, para optimizar esta experiencia. En lo que respecta a este primer año, los resultados han sido más que alentadores. De un total de 61 estudiantes solo $26(42,6 \%)$ realizaron el periodo de acompañamiento diciembre-febrero en el cual se trabajaron contenidos a recuperar sobre materias en las que no alcanzaron lo necesario para aprobarlas. Así, se trabajó en aquel periodo para fortalecer contenidos y afianzar el proceso de aprendizaje sobre los mismos, evitando instancias devaluatorias como resultan los exámenes finales para garantizar un mejor acercamiento con los/as estudiantes.

\section{Reflexiones finales}

En este trabajo reflexionamos acerca del proyecto y la implementación de una Secundaria Técnica impulsada por el Colegio Nacional "Rafael Hernández"y la Facultad de Ingeniería de la UNLP. Esta iniciativa propone repensar el sistema pregrado y crear otras formas de pasaje entre niveles educativos que contemplen las especificidades del contexto, las problemáticas emergentes y las complejidades del tránsito abrupto de una instancia a la otra. La escuela plantea una modalidad orientada al Saneamiento y Gestión Ambiental, proyecto que surge para dar respuesta a múltiples problemáticas sociales, económicas y 
culturales que muchos/as estudiantes enfrentan en el pasaje entre niveles educativos y en el tránsito por cada uno de ellos.

La experiencia es novedosa, como dijimos, por ser la primera de esta índole en muchos sentidos. No existía previamente formación técnico profesional en el Colegio Nacional "Rafael Hernández". Invita a pensar así, nuevas estrategias de enseñanza, objetivos específicos y un accionar conjunto de la UNLP que resulta muy enriquecedor no solamente para los/as estudiantes y la comunidad educativa de la UNLP, sino también para aquellos territorio que serán beneficiados por los resultados de esta formación técnica, objetivo principal de la experiencia de este secundario. De allí la relevancia en consolidar la iniciativa y garantizar la permanencia de los/as estudiantes, fortaleciendo también la especificidad de la formación.

Además, surge como una política de intervención y gestión en la inclusión de una multiplicidad de actores/as en el entramado relacional de la UNLP, garantizando la articulación de ambas unidades académicas así como de la Universidad en general. Se busca así una lectura de problemáticas educativas pensada desde diferentes dimensiones, que permita generar una propuesta de gestión y de abordaje de aquellos disparadores que surgen del balance realizado sobre dichos problemas. El proyecto incluye el interés por mejorar la calidad de la enseñanza apuntando a prácticas de retención e inclusión escolar 
y prácticas de innovación de enseñanza desde una perspectiva territorial, situada y contextual.

Tiene como propósito responder a la demanda de la sociedad, promoviendo la equidad social, custodiando la igualdad de oportunidades y mejorando la calidad de la oferta pública en la enseñanza de pregrado. Para garantizar estas premisas se pusieron en ejercicio coordinaciones y articulaciones entre ambas unidades académicas así como dentro del propio Colegio, que fortalecían el acompañamiento a los/as ingresantes, así como garantizaban condiciones necesarias para su continuidad. Generar condiciones alimentarias, transporte, útiles, acompañamiento del DOE, de docentes y preceptores/ as así como de la gestión en general, garantizó las mejores condiciones posibles para que estos estudiantes no solo pudiesen desarrollarse en las materias sino también habitaran de la mejor manera la institución, apropiándose del espacio.

Los resultados fueron más que alentadores, en reuniones y comentarios, padres, madres, tutores/ as y jóvenes manifestaron su agradecimiento y su felicidad por la experiencia en la que se habían embarcado, por la presencia de la institución y la serie de herramientas dispuestas a atender las distintas necesidades para poder optimizar la experiencia escolar. Pero además, vemos esto en los números ya que hacia el 2020 continuarán el 100\% de los/as estudiantes que ingresaron en primer año en el 2019. Dentro de ellos, están aquellos/as que no alcanzaron el mínimo para aprobar alguna asignatura con 
los que se trabajó de manera conjunta durante algunos meses para garantizar un proceso socio-educativo más integral.

Si bien los desafíos son múltiples y queda mucho por hacer, la experiencia muestra que el trabajo coordinado e integral dentro del Colegio y con otras unidades académicas de la UNLP puede traer grandes resultados. Cuando decimos grandes, no solo nos referimos a la masividad sino más bien a una experiencia que pone en debate múltiples problemáticas y plantea un desafío para la comunidad de la UNLP en general. Por esto, creemos que es necesario seguir este camino, potenciar herramientas y compartir experiencias para fortalecer las intervenciones y articulaciones de la UNLP en general, y del Secundario Técnico en particular. El proceso de inclusión y de engrasación educativa es un desafío de toda la comunidad académica y se convierte, así, en una política educativa importantísima.

\section{Bibliografía}

- Acosta, F. (2011) "La educación secundaria en foco: análisis de políticas de inclusión en Argentina, Canadá, Chile y España”, IIPE-UNESCO, Buenos Aires. Acosta, Felicitas (2011). Escuela secundaria y sistemas educativos modernos: análisis histórico comparado de la dinámica de configuración y expansión en países centrales y en la argentina. Revista HISTEDBR On-line, Campinas, n.42, p. 3-13 
- Auyero, Javier (1993) Otra vez en la vía. Notas e interrogantes sobre la juventud de sectores populares, Buenos Aires: Espacio Editorial.

- Beatriz Andrea Rengif; Liliana Quitiaquez Segura; Francisco Javier Mora Córdoba (2012) La educación ambiental una estrategia pedagógica que contribuye a la solución de la problemática ambiental en Colombia. XII Coloquio Internacional de Geocritica. Bogotá.

- Bracchi, C; Gabbai, I; Paulozzo, M y Hernandez, M. (2010) "Políticas de educación secundaria para adolescentes y jóvenes de la Provincia de Buenos Aires. La implementación de la escuela secundaria obligatoria”. Congreso Iberoamericano de Educación. Metas 2021. Buenos Aires. 13, 14 y 15 de Septiembre de 2010. -

- Chávez, Mariana (2010) Jóvenes, territorios y complicidades. Una antropología de la juventud urbana. Espacio Editorial, Buenos Aires, 2010.

- Dávila Oscar, Felipe Ghirardo y Carlos Medrano (2006): Los desheredados. Trayectorias de vida y nuevas condiciones juveniles. Valparaíso: Ediciones CIDPA.

- Dirección General de Cultura y Educación (2011) "El Régimen Académico para la Educación Secundaria”. Documento para la Primera Jornada Institucional 2011. 
- Dirección Provincial de Planeamiento (2010) "Relevamiento de alumnos con escolaridad en riesgo. Educación secundaria común. Año 2010”. Documento de circulación interna. Subsecretaría de Educación. DGCyE.

- Dirección Provincial de Planeamiento y Dirección Provincial de Educación Secundaria (2012) "Resultados de las evaluaciones de febrero y marzo en escuelas secundarias de la provincia de Buenos Aires (2011/2012). Impacto de las comisiones evaluadoras adicionales y situación de los alumnos de 6to año y titulados (2011/2012)". Documento de circulación interna. Dirección General de Cultura y Educación.

- Dubet, François (2011) Repensar la justicia social. Contra el mito de la igualdad de oportunidades. Buenos Aires: Siglo XXI.

- Dubet, François y Martuccelli, Danilo (1998) En la escuela. Sociología de la experiencia escolar. Barcelona: Losada.

- Duschatzky, Silvia (1999) La escuela como frontera. Buenos Aires: Paidós. Fridman, D. (2013) "Un recorrido conceptual sobre los procesos de socialización en la escuela secundaria argentina. De las normas como imposición a las normas como construcción", en CD de 20 congreso uruguayo de sociología "Desigualdades Sociales y Políticas Públicas en el Uruguay de hoy". Montevideo, 10, 11 y 12 de julio de 2013. ISBN: 978-9974-0-0956-1. 
- Frigerio, G.; Poggi, M. y Tiramonti, G. (1992) Las instituciones educativas. Cara y ceca. Elementos para su comprensión. Buenos Aires: Troquel- FLACSO. Giordano, C. J., Tauber, F., Caminos Lagorio, C., Delucchi, D., Marengo, R., Martino, H., Molteni, M. y Sánchez Arrabal M. B. (2016). Políticas de Planeamiento en la Educación Superior: presupuesto, planes estratégicos y metas educativas globales [archivo PDF]. Recuperado de: http://sedici.unlp.edu.ar/handle/10915/69716

- Hobsbawm, E. J. (2006). La era de la revolución, 1789-1848.

- Huertas F. Planificar para gobernar: El método PES. Entrevista a Carlos Matus. Buenos Aires: Universidad nacional de La Matanza; 2006.

- Karolinski, M. (2006) Lo público y lo privado en educación: un recorrido por las percepciones de los sindicatos docentes sobre los Bachilleratos Populares de jóvenes y adultos [on line] $5^{\circ}$ Jornadas de Jóvenes Investigadores del Instituto Gino Germani, UBA, http://webiigg.sociales.uba.ar/iigg/jovenes investigadores/5jornadasjove nes/EJE11/Karolinsk i.pdf . Visitado en septiembre de 2015.

- Karolinski, M. y Maañón, M. I. (2013) "Estado, educación y organizaciones sociales: aportes para el debate sobre "la gestión social" en la Provincia de Buenos Aires". En C. Abdo Ferez, M. Galvani, D. Pierbattisti y M. C. Rodríguez (Comp.) Nuevos horizontes en la 
investigación social. Buenos Aires: CLACSO; Instituto de Investigaciones Gino Germani, pp. 203- 228.

- KESSLER, Gabriel (2002) La experiencia escolar fragmentada. UNESCO.

- Korinfeld, D. (2013) "Espacios e instituciones suficientemente subjetivados". En

- D. Korinfeld, D. Levy y S. Rascovan, Entre adolescentes y adultos en la escuela. Puntuaciones de una época. Buenos Aires: Paidós.

- Krichesky, M. (Coord.) (2007) Escuelas de Reingreso. Miradas de directores, docentes y alumnos. Buenos Aires: Gobierno de la Ciudad de Bs. As.

- Kriger, M. (2012) (Comp.) Juventudes en América Latina: Abordajes multidisciplinares sobre identidades, culturas y políticas del siglo XX al siglo XXI, del cual es compiladora, Buenos Aires, Ed. CAICYT CONICET.

- LEY 24.521 DE EDUCACION SUPERIOR

http://www.secgral.unsl.edu.ar/wp-content/uploads/docs/Ley-24521-de-E ducacion-Superior.pdf

- LEY DE EDUCACIÓN NACIONAL. Ley 26.206

http://servicios.infoleg.gob.ar/infolegInternet/anexos/120000-124999/123 542/no rma.htm 
- Matus C. Los 3 cinturones del gobierno: gestión, organización y reforma. Caracas: Fondo Editorial Altadir; 1997. p. 5-53.

- Meirieu Philippe; La opción de educar: Etica y Pedagogía, Editorial Octaedro, Madrid, España, 2001.

- Nuñez y Litichever (2015) Radiografías de la experiencia escolar. Ser joven(es) en la escuela. Grupo Editor Universitario. CLACSO.

- PENNAC, Michel (2008) Mal de escuela. Montevideo: Mondadori

- Resolución $N^{\circ} 587 / 11$. Régimen Académico para la Educación Secundaria.

- Rizo, M. y Romeu, V. (2018). Investigación-acción-participativa y comunicación intercultural. Relato de una experiencia de investigación con estudiantes de dos universidades de la ciudad de México. Razón y palabra, 65 .

- Sendon, María Alejandra. "Trayectorias escolares: una aproximación metodológica". Documento on line. Accesible en: http://www.mapaeducativo.edu.ar/Atlas/Trayectorias-Escolares\#_ftnref3

- Spinelli, Hugo. El proyecto político y las capacidades de gobierno. Salud Colectiva, vol. 8, núm. 2, mayo-agosto, 2012, pp. 107-130. Universidad Nacional de Lanús, Buenos Aires, Argentina. 
- Tauber, F. (2005). La universidad y sus desafíos. Revista de la $\begin{array}{lllll}\text { Universidad, } & 1 & \text { (33), } & \text { 49-53. ISSN: } & \text { 0041- }\end{array}$ http://sedici.unlp.edu.ar/handle/10915/68751

- Tauber, Fernando: "Pensar la Universidad" Proyecto Institucional de la UNLP $2018-2022$.

- Tenti Fanfani Emilio; CULTURAS JUVENILES Y CULTURA ESCOLAR, UNIVERSIDAD PEDAGÓGICA NACIONAL, No. 40 - 41 AÑO 2000 .

- Terigi Flavia (2007); LOS DESAFÍOS QUE PLANTEAN LAS TRAYECTORIAS

- ESCOLARES, FUNDACIÓN SANTILLANA, III Foro Latinoamericano de Educación Jóvenes y docentes. La escuela secundaria en el mundo de hoy.

- Tiramonti, Guillermina y otros (2007). Nuevos formatos escolares para promover la inclusión educativa. Un estudio de caso: la experiencia Argentina. Informe final. FLACSO, Bs. As. Disponible en: http://educacion.flacso.org.ar/files/proyectos/pdf/informe formatos escol ares.pdf

- Vommaro, P. (2015). Juventudes y políticas en la Argentina y en América Latina. Ediciones del Aula Taller. 